\title{
The Mediating Role of Depressive Symptoms, Hopelessness, and Perceived Burden on the Association Between Pain Intensity and Late-Life Suicide in Rural China: A Case-Control Psychological Autopsy Study
}

\section{Jiali Wang}

Central South University https://orcid.org/0000-0002-3640-5910

\section{Zhenyu Ma}

Guangxi Medical University

\section{Cunxian Jia}

Shandong University

\section{Guojun Wang}

Shenzhen Kangning Hospital

\section{Liang Zhou ( $\square$ liangzhou_csu@vip.163.com )}

Brain Hospital of Guangzhou Medical University (Guangzhou Huiai Hospital) https://orcid.org/00000003-3207-7419

\section{Research article}

Keywords: suicide, older adults, depressive symptoms, hopelessness, perceived burden, pain intensity

Posted Date: August 21st, 2020

DOl: https://doi.org/10.21203/rs.3.rs-56436/v1

License: (c) (i) This work is licensed under a Creative Commons Attribution 4.0 International License. Read Full License

Version of Record: A version of this preprint was published at Frontiers in Psychiatry on December 13th, 2021. See the published version at https://doi.org/10.3389/fpsyt.2021.779178. 


\section{Abstract}

Background: Few studies have investigated the roles of psychosocial factors such as depressive symptoms and hopelessness on the relationship between pain and suicide with inconsistent results. The study aimed to analyze the impact of pain intensity on suicide death and to estimate the degree to which depressive symptoms, hopelessness, and perceived burden may explain the association in Chinese rural elderly.

Methods: Using a 1: 1 matched case-control design, data were collected from 242 elderly suicide cases and 242 living community controls by psychological autopsy method in rural China, including demographic characteristics, pain intensity, depression, hopelessness, perceived burden, physical diseases, and social support. Conditional logistic regression was used to assess the association between pain intensity and completed suicide. Mediation analysis using the KHB method was applied to explore the mediation effects from depressive symptoms, hopelessness, and perceived burden.

Results: The result of multivariable logistic regression showed that unemployment (odds ratio $(O R)=5.06$, 95\% confidential interval (Cl): 1.76-14.49), higher levels of hopelessness (OR=7.72, 95\% Cl: 3.49-17.10), depressive symptom ( $\mathrm{OR}=15.82,95 \% \mathrm{Cl}$ : 4.53- 55.25), and severe pain ( $\mathrm{OR}=3.46,95 \% \mathrm{Cl}$ : 1.31- 9.13) were significantly associated with elevated suicide risk in older people in rural China. Depressive symptoms, hopelessness, and perceived burden significantly mediated $43.71 \%$ of the pain-suicide association ( $P=$ 0.020 ), with $17.39 \%$ due to depressive symptoms, $17.63 \%$ due to hopelessness, and $8.69 \%$ due to perceived burden.

Conclusions: Regular screening of pain, depressive symptoms, hopelessness, and perceived burden using simple but sensitive questions or scales for older adults with pain are vital for the prevention and early detection of suicide risk in Chinese rural areas. Moreover, the importance of pain management and psychological interventions targeted on depressive symptoms and hopelessness should be emphasized.

\section{Background}

Though the suicide rate in China has declined rapidly, the elderly population increasingly contributes to the overall burden of suicide(1). The average annual suicide rate among old adults was estimated to be 6.5 -fold higher than the rate of the population under 65 , and the rate in rural older adults was two times higher than that in urban elderly(2). Suicide among older adults in rural China remains a critical public health and mental health problem. Identification potential risk factors for this vulnerable population is of paramount significance to design targeted suicide preventions. 
Pain is highly prevalent, costly, and frequently disabling in later life.(3) Almost $30 \%$ of U.S. older adults and $37.1 \%$ (34.1-40.3\%) noninstitutionalized older adults in Hong Kong experienced persisting pain (4, 5). Many older adults suffer silently and needlessly, resulting in severely diminished functional ability(3), interference in daily and social activities (5), and an elevated negative feeling. Previous evidence suggested that pain conditions were associated with an elevated risk of suicidal behaviors(6-8).

Depression has been found to be associated with suicidality in individuals with pain $(9,10)$. One study observed that pain-suicide association reduced when controlling for psychiatric diagnoses, including depression (7), indicating that pain might be linked to suicidality via mechanisms at least related to depression.

Hopelessness has been identified as relevant in the understanding of the physical pain-suicidality link (11). A large pan-Canadian study found that patients with higher pain-related helplessness and greater pain magnification were more likely to present suicidal ideation. Besides, patients with hope towards medical cure had less suicidal thoughts (9). Higher hopelessness was associated with a higher pain threshold, which correlated with less fearlessness of death $(12,13)$.

The interpersonal theory of suicide proposed that perceived burdensomeness is a precursor to a desire to commit suicide (14). Individuals suffering from pain may feel that they are creating a burden on their families. One study supported the theory by showing that perceived burdensomeness significantly predicted suicidal ideation in people with chronic pain (15). Moreover, the feeling of being a burden may be exacerbated by depression and hopelessness, finally increasing the risk of carrying out a suicidal act (16).

Though the pain-suicidality link has been reported, the mechanism may be complicated. Limited studies investigated the mediation effect of psychological factors, including depression, anxiety, and sleep problem on pain and suicidality link with inconsistent results (17-19). Moreover, most studies on the pain-suicidality association focused on suicide ideation and suicide attempts.

Using a matched case-control psychological autopsy study design, the goal of the present study was to analyze the link between pain and completed suicide among Chinese rural older adults and to estimate the degree to which depressive symptoms, hopelessness, and perceived burden may explain the association.

\section{Methods}

\section{Sample and Sampling}

A multi-stage stratified cluster sampling method was used to select the study site. The first step was the selection of the province. Thirty-one provinces in mainland China were stratified into three levels according to the gross domestic product per capita. Shandong province, Hunan province, and Guangxi province were chosen from the rank 1-10, 11-20 and 21-31, respectively. The second step was the 
selection of rural counties from the selected provinces. Counties were stratified into three levels based on per capita income in each province. One county in each stratum from Shandong and Hunan, and two counties in each stratum from Guangxi (as the population sizes of the counties in Guangxi were smaller than those in the other two) were selected using a simple random sampling performed by a computer algorithm. A total of 12 counties from the three selected provinces were included in this study. The study was conducted from June 2014 to September 2015.

In each county, suicide cases of individuals aged 60 and above in rural areas(villages) were collected consecutively based on the death certification system. A senior suicidologist briefly trained all village doctors and local public health workers involved in death certification on how to determine a suicide death. They were required to report all elderly suicide deaths, as well as possible cases that they could not determine, to the local Centers for Disease Control and Prevention. Trained investigators finally determined the manner of death after all available, relevant information had been collected.

Living comparisons were 1:1 matched with the suicide cases in gender, birth year ( \pm 3 years), and living location. Whenever a suicide case was identified, the investigators would list and enumerate all older adults matching gender and birth year in the same village. One living comparison was then selected from the list randomly using a computer program. The investigators expanded the search to the closest village if no appropriate control individuals were available in the same village. A total of 242 suicide victims and 242 living comparisons were enrolled in this study.

\section{Procedures of interview}

For each suicide case and living control, we selected two informants. Generally, the first informant was a next of kin who lived with the suicide case or the living control. The second informant was a friend, neighbor, or remote relative. Interviews with the informants of suicide cases were scheduled 2 to 6 months after the death, while interviews with informants of living comparisons were conducted as soon as the participants and their informants were identified. Each informant was interviewed separately by one trained fieldworker, and the interviews lasted 90 min on average. The details of the subjects' selection and interview procedures have been described in previous publications (20).

All procedures were carried out in accordance with the latest version of the Declaration of Helsinki. The study was approved by the Institutional Review Boards of the Central South University, Shandong University, and Guangxi Medical University. The aim and procedure of the research were explained to all participants. Written informed consent was obtained from participants in the living control group and all the informants of suicide cases and living comparisons after the nature of the procedures had been fully explained.

\section{Measures}

\section{Socio-demographic characteristics}


We collected socio-demographic characteristics, including age, gender, marital status, employment status, education level, annual family income.

\section{Pain intensity}

We used an 11-point visual analog scale to assess the self-perceived pain intensity. First, participants were asked, "Did you/he/she feel any pain (including any physical pain or pain with unknown reasons) before death/ investigation?" Participants who endorsed "yes" were asked to report their pain intensity by circling "the one number that describes your/ his or her pain on the average," using 0 (no pain) to 10 (the worst pain ) scale(21). We categorized pain intensity into four groups: 0 means without pain, 13 mild pain, 46 moderate pain, and 710 severe pain. Since the number of participants in the mild pain group was small (34(14.05\%) in the suicide cases and 31(12.81\%) in the living controls), we combined mild and moderate pain into a single category called "mild to moderate pain."

\section{Physical diseases}

We used a question asking, "Did you/he/she have diagnosed physical diseases" to investigate whether having physical diseases before death/investigation. 0 means without physical disease, 1 means with one or more physical diseases.

\section{Social support}

We used the 23-items Duke Social Support Index (DDSI) to measure social support in the last week before suicide/ investigation. A total score ranges from 11 to 45 , and a higher score indicates higher social support. DSSI has been used in a previous psychological autopsy study in China and showed satisfactory reliability and validity(22). Social support was dichotomized into two groups based on the median score of DSSI.

\section{Perceived burden}

We used the question that "Did you/he/she feel that caring you/he/she bring mental or physical burden to your/his/her family?" to rank the perceived burden level. 1 means "no perceived burden," 4 means "heavy perceived burden." Perceived burden was categorized into two groups (1 means without perceived burden; 2-4 means with perceived burden).

\section{Depressive symptoms}

We used the Geriatric Depression Scale (GDS-30) to assess the depressive symptoms in the last week before death/ investigation(23). A higher score means more severe depressive symptoms. A cut-off score of 10 and 20 represents mild and severe levels of depressive symptoms, respectively. The GDS-30 has shown adequate properties in the Chinese elderly(23). In the conditional logistic regression, GDS score was categorized into two groups: 0-10 means without depressive symptoms; $11-30$ means with depressive symptoms.

\section{Hopelessness}


We used the BHS-4 to measure hopelessness in the last week before death/ investigation(24). It consists of four items relevant to success, dark future, breaks, and faith. Each item is rated on a 5-point scale ranging from 1 (strongly agree) to 5 (strongly disagree). A total score ranges from 4 to 20, and a higher score represents a higher level of hopelessness(24). Severity of hopelessness was categorized into two groups according to the median of BHS score.

\section{Data combination}

We combined the information provided by the two informants as the proxy data for each suicide case and living control. Proxy data was used for analysis. For socio-demographic data, we relied on the information provided by the first informant. Regarding psychosocial factors, we used answers that were hypothetically associated with an elevated risk of suicide, considering any targeting symptoms or behaviors might exist as long as any informant may have observed $(20,25)$. Thus, the higher score of pain intensity, perceived burden, and BHS-4 was used; similarly, a positive answer of an item of GDS-30 was used if one of the two informants reported positive.

Our previous studies had proved the validity of proxy data in in psychological autopsy study $(26,27)$. We also compared the proxy-based information of pain intensity with the data reported by the subjects themselves in the living control group. The subject-proxy agreement was fair with an ICC value of 0.504 (95\% Cl: $0.361,0.615, P<0.001)$, and it supports the validity of the proxy data on pain intensity.

\section{Statistical analyses}

Descriptive analysis, chi-squared tests for categorical variables, and Student's t-tests for continuous variables were used to describe and compare the socio-demographics, pain intensity, perceived burden, hopelessness, and depressive symptoms of suicide cases and living controls. Conditional logistic regression (backward stepwise selection) was used to assess the association between pain intensity and completed suicide. Covariates adjusted: marital status, employment status, education level, annual family income, physical disease, social support, depressive symptoms, hopelessness, and perceived burden.

Mediation analysis was conducted to assess the specific contribution of depressive symptoms, hopelessness, and perceived burden in the pain-suicide association. The KHB method can be used to decomposes total effect ( the effect of pain on suicide without adjustment of mediators) into direct effect ( the effect of pain on suicide) and indirect effects (the mediation effects from depressive symptoms, hopelessness and perceived burden) in conditional logistic regression(28, 29). Moreover, the confounding percentage to total effect due to mediators, and disentangle contribution of each mediator can be calculated. Marital status, employment status, education level, annual family income, physical disease, social support were controlled in the mediation analysis. The mediation analysis was performed with the khb command. Entering method, rather than backward stepwise selection was used in mediation analysis since the khb command is not supported by stepwise method. All analysis was performed using Stata 16.0 (College Station, Texas 77845 USA). The level of statistical significance was set at $P<0.05$. 


\section{Results}

\section{Characters of suicide cases and living controls}

We investigated 242 suicide cases and 242 living controls. The mean age was $74.4 \pm 8.2$ years, and $55.8 \%$ were male among the suicide cases. The mean age was $74.1 \pm 8.2$ years, and $55.8 \%$ were male among living controls. Compared with the living controls, the suicide cases tended to be not currently married, be unemployment, with physical disease (all $P$ values $<0.05$ ). Suicide cases reported higher pain intensity, more perceived burden, higher level of depressive symptoms and hopelessness, and lower level of social support than living controls (all $P$ values $<0.01$ ). Descriptive data for study variables are presented in Table 1. 
Table 1

Characters of suicide cases and living controls

\begin{tabular}{|c|c|c|c|c|}
\hline Variables & $\begin{array}{l}\text { Suicide cases }(\mathrm{n}= \\
242)\end{array}$ & $\begin{array}{l}\text { Living controls }(n= \\
\text { 242) }\end{array}$ & $\mathrm{z} / \mathrm{t} / \mathrm{\chi}^{2}$ & $P$ \\
\hline Age [mean (S.D.)] & $74.4 \pm 8.2$ & $74.1 \pm 8.2$ & 0.51 & 0.609 \\
\hline \multicolumn{5}{|l|}{ Gender } \\
\hline Male & $135(55.8)$ & $135(55.8)$ & & \\
\hline Female & $107(44.2)$ & $107(44.2)$ & & \\
\hline Marital status & & & 19.89 & $\begin{array}{l}< \\
0.001\end{array}$ \\
\hline Currently married & $122(50.4)$ & $170(70.2)$ & & \\
\hline Not currently married & $120(49.6)$ & $72(29.8)$ & & \\
\hline Employment status & & & 8.27 & 0.016 \\
\hline Employed & $41(16.9)$ & $61(25.2)$ & & \\
\hline Unemployed & $194(80.2)$ & $167(69.0)$ & & \\
\hline Retired & $7(2.9)$ & $14(5.8)$ & & \\
\hline Education & & & 1.92 & 0.383 \\
\hline Blow primary school & $111(45.9)$ & $96(39.7)$ & & \\
\hline Primary school or above & $105(43.4)$ & $116(47.9)$ & & \\
\hline Above primary school & $26(10.7)$ & $30(12.4)$ & & \\
\hline Physical disease & & & 18.52 & $\begin{array}{l}<.001 \\
0.00\end{array}$ \\
\hline Without & $40(16.53)$ & $81(33.47)$ & & \\
\hline Any & 202(83.47) & $161(66.53)$ & & \\
\hline Social support [mean (S.D.)] & $22.9 \pm 6.0$ & $27.5 \pm 6.8$ & 7.87 & $\begin{array}{l}<.001 \\
0.001\end{array}$ \\
\hline Lower group $(\leq 25)$ & $166(68.60)$ & $95(39.26)$ & 41.92 & $\begin{array}{l}<.001 \\
0.00\end{array}$ \\
\hline Higher group (> 25) & $76(31.40)$ & $147(60.74)$ & & \\
\hline Pain intensity & $4.0 \pm 3.8$ & $2.5 \pm 3.0$ & 4.85 & $\begin{array}{l}< \\
0.001\end{array}$ \\
\hline None(0) & $93(38.43)$ & $124(51.24)$ & 45.18 & $\begin{array}{l}<.001 \\
0.001\end{array}$ \\
\hline
\end{tabular}




\begin{tabular}{|c|c|c|c|c|}
\hline Variables & $\begin{array}{l}\text { Suicide cases }(\mathrm{n}= \\
\text { 242) }\end{array}$ & $\begin{array}{l}\text { Living controls }(n= \\
242)\end{array}$ & $z / t / \chi^{2}$ & $P$ \\
\hline Mild to moderate $(1-6)$ & $59(24.38)$ & $91(37.60)$ & & \\
\hline Severe $(7-10)$ & $90(37.19)$ & $27(11.16)$ & & \\
\hline Perceived burden [mean (S.D.)] & $2.1 \pm 1.3$ & $1.3 \pm 1.1$ & 7.43 & $\begin{array}{l}<.001 \\
0.001\end{array}$ \\
\hline Without $(=1)$ & $72(29.75)$ & $130(53.72)$ & 28.58 & $\begin{array}{l}<.001 \\
0.00\end{array}$ \\
\hline With $(>1)$ & $170(70.25)$ & $112(46.28)$ & & \\
\hline $\begin{array}{l}\text { Depressive symptom [mean } \\
\text { (S.D.)] }\end{array}$ & $21.4(5.9)$ & $9.2(6.4)$ & 21.65 & $\stackrel{<}{0.001}$ \\
\hline Without $(\leq 10)$ & $15(6.20)$ & $156(64.46)$ & 179.78 & $\begin{array}{l}<.001 \\
0.001\end{array}$ \\
\hline With $(>10)$ & $227(93.80)$ & $86(35.54)$ & & \\
\hline Hopelessness [mean (S.D.)] & $14.6(2.5)$ & $9.7(2.7)$ & 20.42 & $\begin{array}{l}<.001 \\
0.001\end{array}$ \\
\hline Lower group $(\leq 12)$ & $50(20.66)$ & $207(85.54)$ & 204.49 & $\begin{array}{l}< \\
0.001\end{array}$ \\
\hline Higher group (> 12) & 192(79.34) & $35(14.46)$ & & \\
\hline
\end{tabular}

\section{Logistic regression analysis}

Conditional logistic regression was employed to detect risk factors of suicide completion among older adults in rural China. As shown in Table 2, unemployment (odds ratio $(O R)=5.06,95 \%$ confidential interval ( $\mathrm{Cl}$ ): 1.76-14.49), higher level of hopelessness ( $\mathrm{OR}=7.72,95 \% \mathrm{Cl}: 3.49-17.10)$, depressive symptom ( $\mathrm{OR}=15.82,95 \% \mathrm{Cl}: 4.53-55.25)$, and severe pain $(\mathrm{OR}=3.46,95 \% \mathrm{Cl}: 1.31-9.13)$ were significantly associated with suicide completion. Mild to moderate (OR $=0.54 ; 95 \% \mathrm{Cl}: 0.20-1.43)$ pain was not significantly associated with suicide completion. 
Table 2

Multivariate logistic regression for completed suicide among the rural elderly in China

\begin{tabular}{|c|c|c|c|}
\hline Independent variables & OR & $95 \% \mathrm{Cl}$ & $P$ \\
\hline \multicolumn{4}{|l|}{ Employment status } \\
\hline Employed & 1.00 (ref) & - & - \\
\hline Unemployed & 5.06 & $1.76-14.49$ & 0.003 \\
\hline Retired & 2.13 & $0.25-18.09$ & 0.490 \\
\hline \multicolumn{4}{|l|}{ Hopelessness } \\
\hline Lower group ( $\leq 12$ ) & 1.00 (ref) & - & - \\
\hline Higher group (> 12) & 7.72 & $3.49-17.10$ & $<0.001$ \\
\hline \multicolumn{4}{|l|}{ Depressive symptoms } \\
\hline Without $(\leq 10)$ & 1.00 (ref) & - & - \\
\hline with $(>10)$ & 15.82 & $4.53-55.25$ & $<0.001$ \\
\hline \multicolumn{4}{|l|}{ Pain intensity } \\
\hline None (0) & 1.00 (ref) & - & - \\
\hline Mild to moderate $(1-6)$ & 0.54 & $0.20-1.43$ & 0.213 \\
\hline Severe $(7-10)$ & 3.46 & $1.31-9.13$ & 0.012 \\
\hline \multicolumn{4}{|c|}{$\begin{array}{l}\text { Adjusted for education level, employment, marital status, annual family income, physical disease, } \\
\text { social support, perceived burden, hopelessness, and depressive symptoms. OR, odds ratio; } \mathrm{Cl} \text {, } \\
\text { confidential interval. } \mathrm{R}^{2}=0.7084 \text {. }\end{array}$} \\
\hline
\end{tabular}

\section{Mediation analysis}

The results of the mediation analysis are shown in Table 3. To simplify interpretation of OR, pain intensity was dichotomized as "moderate or less" and "severe" because increased risk of suicide completion was observed only in the older adults with severe pain. Depressive symptoms, hopelessness, and perceived burden significantly mediated the association between pain and completed suicide $(P=0.020)$. The total effect of pain intensity on suicide completion was 1.78 times larger than the direct effect, and $43.71 \%$ of the total effect was due to mediators (indirect effect). In terms of the specific contributions of mediators, depressive symptoms explained $17.39 \%$ of the association between severe pain and suicide completion, hopelessness explained $17.63 \%$, and perceived burden explained $8.69 \%$. Depressive symptoms alone could not significantly mediate pain- suicide association. Nor could hopelessness, or perceived burden. Any combinations of two factors did not have a significant mediational effect (All $P>0.05$ ) (Supplementary materials). 
Table 3

Mediation analysis for the association between pain and completed suicide.

\begin{tabular}{|llll|}
\hline Effects & OR & $95 \% \mathrm{Cl}$ & $\boldsymbol{P}$ \\
\hline Total effect & 14.46 & $4.51-46.32$ & $<0.001$ \\
\hline Direct effect & 4.50 & $1.47-13.73$ & 0.008 \\
\hline Indirect effect & 3.21 & $1.21-8.56$ & 0.020 \\
\hline $\begin{array}{l}\text { Exposure: pain intensity, outcome: completed suicide, mediators: depressive symptoms, } \\
\text { hopelessness, and perceived burden. Covariates: education level, employment, marital status, annual } \\
\text { family income, physical disease, social support. The total effect is 1.78 times larger than the direct } \\
\text { effect, and 43.71\% of the total effect is due to mediators, with 17.39\% due to depressive symptoms, } \\
\text { 17.63\% due to hopelessness, and 8.69\% due to perceived burden. }\end{array}$ \\
\hline
\end{tabular}

\section{Discussion}

As of our knowledge, our study is the first one using suicide completion as the outcome to investigate the mediation effect of and depressive symptoms, hopelessness, and perceived burden on the pain-suicide association and to quantify the individual mediational effect. We find that pain was an independent risk factor of suicide completion in older adults in rural China. Furthermore, our results indicated that depressive symptoms, hopelessness, and perceived burden significantly but partially mediated the painsuicide association.

Previous studies found that patients with pain had increased risk of suicidal outcomes compared with the general population. A large cohort study demonstrated that veterans with self-reported severe pain were more likely to die by suicide than patients experiencing moderate or less pain (HR: $1.33,95 \% \mathrm{Cl}$ : 1.15-1.54) (30). A case-control study among older adults in Canada also found that severe pain (OR: 4.07, 95\% Cl: 2.51-6.59) elevated the risk of suicide death (31). However, the study only considered patients with prescribed pain medication. Consistent with previous studies, the present study found that severe pain was more common in suicide cases than in controls (37.19\% vs. $11.16 \%)$. We proved that older adults with severe pain predicted 3.46 times of suicide completion risk than those without pain. Older adults with severe pain may view suicide as a way to escape from suffering (32). The risk for suicide among the older population with severe pain merits particular attention.

One major finding was that $56.29 \%$ of the total effect of pain intensity on suicide was from pain directly, and perceived burden, hopelessness, and depressive symptoms significantly mediated $43.71 \%$ of the association between pain and suicide. These results were consistent with one recent study proving that depression and anxiety disorders had mediational effects on the relationships between pain-suicidal behaviors(17). Though this study only used suicide ideation and suicide attempt as outcomes, the study used a large, representative sample of the English adult population to prove the partially mediation effect of depression. However, several studies found different results that pain- suicide ideation relationship was fully mediated by depression $(18,33)$. A possible reason for this discrepancy might be due to the 
different outcomes (suicide completion vs. suicidal ideation/attempt) and different populations across these studies.

Moreover, we measured the individual mediational effect. Depressive symptoms, hopelessness, and perceived burden explained $17.39 \%, 17.63 \%$, and $8.69 \%$ of the total effect of pain intensity on suicide completion, respectively. Many studies suggested that hopelessness significantly predicted suicidal behaviors. A meta-analysis of longitudinal studies found that hopelessness significantly predicted suicidal ideation, suicide attempt, and suicide death (34). Moreover, helplessness/ hopelessness in patients with pain has been reported as a significant predictor of suicidal ideation in previous studies ( 9 , $15,35)$. Our results suggest that further investigation should focus more on hopelessness in the painsuicide association.

Consistent with prior evidences (36), we also found the mediating effect of the perceived burden on the pain-suicide association. The interpersonal theory of suicide proposed that thwarted belongingness and perceived burdensomeness were key factors contributing to a suicide desire (14). Perceived burden has been identified as a critical predictor of suicidality in patients with pain $(15,37)$. It is reasonable to expect that the older adults suffering from pain felt that they created a burden to their family. The current finding provided further evidence that the need to consider perceived burdens in older adults with pain in rural areas in suicide prevention.

Previous studies have reported depression as the most common mental illness among suicide decedents $(20,38,39)$. Moreover, higher pain severity was a significant predictor of the onset of depression (40). Our results in line with previous evidence. We further demonstrated that depression partially mediated the impact of pain on suicide.

Interestingly, we found that only depressive symptoms could not mediate the association between pain and suicide completion. Nor did the perceived burden or hopelessness. The relationship between pain and suicide is complex and involves several psychological and psychosocial factors(32). Moreover, those factors might interact with each other, and the interactions may affect the pain-suicidality relationship. A study in back pain patients found that pain intensity showed indirect effects due to pain-related helplessness/ hopelessness on depression (41). Depressive symptoms were found to be a significant predictor of self-perceived burdensomeness in patients with chronic pain. Furthermore, a communitybased study suggested that hopelessness and the interaction between perceived burdens and hopelessness were significantly associated with suicide plans and attempts (42).

Depression, hopelessness, and perceived burden might interact with and exacerbate each other, finally increasing the vulnerability to commit suicide. Though we found the mediators and their respective indirect effects, further researches on the interactions among mediators are needed for a comprehensive understanding of pain-suicide association.

The current study has several limitations. Though psychological autopsy is a widely used method to explore the risk factors of completed suicide, the validity of data provided by proxy informants is a 
concern. However, one previous study had proved that subject-proxy concordance for the GDS was fair (ICC $=0.590)$ in the living controls $(26)$. Second, we measured pain with a single question concerning the intensity of pain without data on the location, type, and duration. More detailed questions on pain might be better for the comprehensive understanding between pain and suicide completion. However, most research generally examines only a limited number of types or locations of pain. Moreover, a review had suggested that pain itself, irrespective of which type or location, was a determinant of suicidality based on abundant evidence (43). Furthermore, it seems no strong link between pain duration and suicidality. As the duration of suffering pain increases, suicidal behaviors may be less related to pain symptoms (43). Also, prior studies had demonstrated that sleep disturbance was a mediator in the pain-suicidality link (19). Nevertheless, we did not include information on sleep quality. Further research is warranted to examine this possibility. Finally, we estimated the pain-suicide death association and mediational effects in older adults in rural China. The results should be interpreted with caution.

\section{Conclusions}

Despite these limitations, our study added to a growing body of evidence indicating that pain independently increased the risk of committing suicide and that perceived burden, hopelessness, and depressive symptoms partially mediated the pain-suicide association. Given the undertreated pain in late life and backward mental health services in rural areas, regular screening of pain, depressive symptoms, hopelessness, and perceived burden using simple but sensitive questions or scales for older adults with pain is vital for the prevention and early detection of suicide risk. Furthermore, treatment of pain and psychological interventions for alleviating depressive symptoms and feelings of hopelessness are critical for suicide prevention.

\section{Declarations}

\section{Declarations}

\section{Ethics approval and consent to participate}

All procedures were carried out in accordance with the latest version of the Declaration of Helsinki. The study was approved by the Institutional Review Boards of the Central South University, Shandong University, and Guangxi Medical University. Written informed consent was obtained from participants in the living control group and all the informants of suicide cases and living comparisons after the nature of the procedures had been fully explained.

\section{Consent for publication}

Not applicable.

\section{Availability of data and materials}


The dataset used and analyzed during the current study is available from the corresponding author on reasonable request.

\section{Competing interests}

The authors declare that they have no competing interests.

\section{Funding}

This work was supported by the American Foundation for Suicide Prevention [Grant No. SRG-0-169-12]; and Science and Technology Plan Project of Guangdong Province [No.2019B030316001].

\section{Authors' contributions}

CJ, ZM, and LZ contributed to the study design; GW contributed to data collection; JW: conducted data analysis and drafted the article; LZ provided substantial editorial input in the drafting of the manuscript. All authors read and approved the final manuscript.

\section{Acknowledgements}

We would like to thank all fieldworkers and participants of this study.

\section{References}

1. Li M, Katikireddi SV. Urban-rural inequalities in suicide among elderly people in China: a systematic review and meta-analysis. Int J Equity Health. 2019;18(1):2.

2. Zhong BL, Chiu HF, Conwell Y. Rates and characteristics of elderly suicide in China, 2013-14. J Affect Disord. 2016;206:273-9.

3. Brown ST, Kirkpatrick MK, Swanson MS, McKenzie IL. Pain experience of the elderly. Pain Manag Nurs. 2011;12(4):190-6.

4. Dahlhamer J, Lucas J, Zelaya C, Nahin R, Mackey S, DeBar L, et al. Prevalence of Chronic Pain and High-Impact Chronic Pain Among Adults - United States, 2016. MMWR Morb Mortal Wkly Rep. 2018;67(36):1001-6.

5. Wong WS, Fielding R. Prevalence and characteristics of chronic pain in the general population of Hong Kong. J Pain. 2011;12(2):236-45.

6. Calati R, Bakhiyi $C L$, Artero $S$, llgen $M$, Courtet $P$. The impact of physical pain on suicidal thoughts and behaviors: Meta-analyses. J Psychiatr Res. 2015;71:16-32.

7. Ilgen MA, Kleinberg F, Ignacio RV, Bohnert AS, Valenstein M, McCarthy JF, et al. Noncancer pain conditions and risk of suicide. JAMA psychiatry. 2013;70(7):692-7.

8. Park MJ, Choi KW, Na EJ, Hong JP, Cho MJ, Fava M, et al. Multiple types of somatic pain increase suicide attempts and depression: A nationwide community sample of Korean adults. Compr Psychiatry. 2019;90:43-8. 
9. Racine M, Sanchez-Rodriguez E, Galan S, Tome-Pires C, Sole E, Jensen MP, et al. Factors Associated with Suicidal Ideation in Patients with Chronic Non-Cancer Pain. Pain Med. 2017;18(2):283-93.

10. Bromberg MH, Law EF, Palermo TM. Suicidal Ideation in Adolescents With and Without Chronic Pain. Clin J Pain. 2017;33(1):21-7.

11. Tang NK, Crane C. Suicidality in chronic pain: a review of the prevalence, risk factors and psychological links. Psychol Med. 2006;36(5):575-86.

12. Orbach I, Mikulincer M, King R, Cohen D, Stein D. Thresholds and tolerance of physical pain in suicidal and nonsuicidal adolescents. J Consult Clin Psychol. 1997;65(4):646-52.

13. Ribeiro JD, Witte TK, Van Orden KA, Selby EA, Gordon KH, Bender TW, et al. Fearlessness about death: the psychometric properties and construct validity of the revision to the acquired capability for suicide scale. Psychol Assess. 2014;26(1):115-26.

14. Van Orden KA, Witte TK, Cukrowicz KC, Braithwaite SR, Selby EA, Joiner TE. Jr. The Interpersonal Theory of Suicide. Psychol Rev. 2010;117(2):575-600.

15. Wilson KG, Heenan A, Kowal J, Henderson PR, McWilliams LA, Castillo D. Testing the Interpersonal Theory of Suicide in Chronic Pain. Clin J Pain. 2017;33(8):699-706.

16. Fishbain DA, Lewis JE, Gao J. The pain suicidality association: a narrative review. Pain Med. 2014;15(11):1835-49.

17. Jacob L, Maria Haro J, Koyanagi A. The association between pain and suicidal behavior in an English national sample: The role of psychopathology. J Psychiatr Res. 2018;98:39-46.

18. Lewcun B, Kennedy TM, Tress J, Miller KS, Sherker J, Sherry DD. Predicting suicidal ideation in adolescents with chronic amplified pain: The roles of depression and pain duration. Psychol Serv. 2018;15(3):309-15.

19. Owen-Smith AA, Ahmedani BK, Peterson E, Simon GE, Rossom RC, Lynch FL, et al. The Mediating Effect of Sleep Disturbance on the Relationship Between Nonmalignant Chronic Pain and Suicide Death. Pain Practice. 2019;19(4):382-9.

20. Zhou L, Wang G, Jia C, Ma Z. Being left-behind, mental disorder, and elderly suicide in rural China: a case-control psychological autopsy study. Psychological medicine. 2019;49(3):458-64.

21. Jensen MP, Turner JA, Romano JM, Fisher LD. Comparative reliability and validity of chronic pain intensity measures. Pain. 1999;83(2):157-62.

22. Zhang J, Conwell Y, Wieczorek WF, Jiang C, Jia S, Zhou L. Studying Chinese suicide with proxy-based data: reliability and validity of the methodology and instruments in China. J Nerv Ment Dis. 2003;191(7):450-7.

23. He X, Xiao S, Zhang G. Reliability and Validity of the Chinese Version of Geriatric Depression Scale:A Study in A Population of Chinese Rural Community-dwelling Elderly. Chinese Journal of Clinical Psychology. 2008;16(5):473-5.

24. Yip PS, Cheung YB. Quick assessment of hopelessness: a cross-sectional study. Health Qual Life Outcomes. 2006;4:13. 
25. Zhang J, Wang Y, Fang L. How to integrate proxy data from two informants in life event assessment in psychological autopsy. BMC Psychiatry. 2018;18(1):115.

26. Niu L, Jia C, Ma Z, Wang G, Yu Z, Zhou L. Validating the Geriatric Depression Scale with proxy-based data: A case-control psychological autopsy study in rural China. J Affect Disord. 2018;241:533-8.

27. Niu L, Jia C, Ma Z, Wang G, Yu Z, Zhou L. The validity of proxy-based data on loneliness in suicide research: a case-control psychological autopsy study in rural China. BMC Psychiatry. 2018;18(1):116.

28. Breen R, Karlson KB, Holm A. Total, Direct, and Indirect Effects in Logit and Probit Models. Sociological Methods Research. 2013;42(2):164-91.

29. Kohler U, Karlson K. KHB: Stata module to decompose total effects into direct and indirect via KHBmethod 2010 [updated revised 24 Feb 2019. Available from: https://ideas.repec.org/c/boc/bocode/s457215.html.

30. Ilgen MA, Zivin K, Austin KL, Bohnert AS, Czyz EK, Valenstein M, et al. Severe pain predicts greater likelihood of subsequent suicide. Suicide Life Threat Behav. 2010;40(6):597-608.

31. Juurlink DN, Herrmann N, Szalai JP, Kopp A, Redelmeier DA. Medical illness and the risk of suicide in the elderly. Arch Intern Med. 2004;164(11):1179-84.

32. Hooley JM, Franklin JC, Nock MK. Chronic pain and suicide: understanding the association. Curr Pain Headache Rep. 2014;18(8):435.

33. Cho S, Hwang H, Lee J-H. Pain intensity and suicidal ideation of people in chronic pain: mediating effects of depression. Soc Behav Pers. 2013;41(3):509-16.

34. Ribeiro JD, Huang X, Fox KR, Franklin JC. Depression and hopelessness as risk factors for suicide ideation, attempts and death: meta-analysis of longitudinal studies. Br J Psychiatry. 2018;212(5):279-86.

35. Racine M, Choiniere M, Nielson WR. Predictors of suicidal ideation in chronic pain patients: an exploratory study. Clin J Pain. 2014;30(5):371-8.

36. Shim E, Song YW, Park SH, Lee KM, Go DJ, Hahm BJ. Examining the Relationship Between Pain Catastrophizing and Suicide Risk in Patients with Rheumatic Disease: the Mediating Role of Depression, Perceived Social Support, and Perceived Burdensomeness. Int J Behav Med. 2017;24(4):501-12.

37. Fishbain DA, Bruns D, Bruns A, Gao J, Lewis JE, Meyer LJ, et al. The Perception of Being a Burden in Acute and Chronic Pain Patients Is Associated with Affirmation of Different Types of Suicidality. Pain Med. 2016;17(3):530-8.

38. Zhang J, Zhou L. A case control study of suicides in China with and without mental disorder. Crisis. 2009;30(2):68-72.

39. Phillips MR, Yang G, Zhang Y, Wang L, Ji H, Zhou M. Risk factors for suicide in China: a national case-control psychological autopsy study. Lancet. 2002;360(9347):1728-36.

40. Gerrits MMJG, van Oppen P, van Marwijk HWJ, Penninx BWJH, van der Horst HE. Pain and the onset of depressive and anxiety disorders. Pain. 2014;155(1):53-9. 
41. Hulsebusch J, Hasenbring MI, Rusu AC. Understanding Pain and Depression in Back Pain: the Role of Catastrophizing, Help-/Hopelessness, and Thought Suppression as Potential Mediators. Int J Behav Med. 2016;23(3):251-9.

42. Kleiman EM, Liu RT, Riskind JH. Integrating the interpersonal psychological theory of suicide into the depression/suicidal ideation relationship: a short-term prospective study. Behav Ther. 2014;45(2):212-21.

43. Racine M. Chronic pain and suicide risk: A comprehensive review. Prog Neuropsychopharmacol Biol Psychiatry. 2018;87(Pt B):269-80.

\section{Supplementary Files}

This is a list of supplementary files associated with this preprint. Click to download.

- Supplementarymaterials.docx 